\title{
Development of a Modified Surgical Technique for Simulating Ischemic Cerebral Cortex Injury in Rats
}

\author{
CHIEN-FU YEH ${ }^{1,2,3 *}$, TUNG-YUEH CHUANG ${ }^{4 *}$, YU-WEN HUNG ${ }^{5}$, MING-YING LAN ${ }^{2,3}$, \\ CHING-HAN TSAI ${ }^{4}$, HAO-XIANG HUANG ${ }^{4}$ and YUNG-YANG LIN ${ }^{1,4,6,7,8}$ \\ ${ }^{1}$ Institute of Brain Science, National Yang-Ming University, Taipei, Taiwan, R.O.C.; \\ ${ }^{2}$ Department of Otorhinolaryngology, National Yang-Ming University, Taipei, Taiwan, R.O.C.; \\ ${ }^{3}$ Department of Otolaryngology-Head and Neck Surgery, Taipei Veterans General Hospital, Taipei, Taiwan, R.O.C.; \\ ${ }^{4}$ Department of Critical Care Medicine, Taipei Veterans General Hospital, Taipei, Taiwan, R.O.C.; \\ ${ }^{5}$ Institute of Cellular and System Medicine, National Health Research Institutes, Miaoli, Taiwan, R.O.C.; \\ ${ }^{6}$ Institute of Physiology, National Yang-Ming University, Taipei, Taiwan, R.O.C.; \\ ${ }^{7}$ Institute of Clinical Medicine, National Yang-Ming University, Taipei, Taiwan, R.O.C.; \\ ${ }^{8}$ Department of Neurology, Neurological Institute, Taipei Veterans General Hospital, Taipei, Taiwan, R.O.C.
}

\begin{abstract}
Background/Aim: Middle cerebral artery occlusion (MCAO) in rodents is an essential animal model for research focusing on ischemic stroke. To date, several kinds of surgical methods for MCAO have been developed and the craniotomy method has the advantage of direct visualization of the middle cerebral artery (MCA). MCAO at a more proximal site produces better surgical results, but it is a more invasive technique. The aim of this study was to evolve the surgical technique for simulating ischemic cerebral cortex injury in rats. Materials and Methods: To approach proximal MCA with a less invasive procedure, a modified surgical technique for MCAO in rats was developed. Besides, rats receiving the modified and conventional method were compared with regard to infarct volume and by behavioral tests. Results: Following craniotomy, we proposed that the inferior edge of the craniotomy should be enlarged with fine forceps. This modified surgical method induces larger infarct volume, significant behavioral impairment and can induce ischemic stroke. Additionally, it does not significantly increase the
\end{abstract}

This article is freely accessible online.

*These Authors contributed equally to this work.

Correspondence to: Yung-Yang Lin, MD, Ph.D., Institute of Brain Science, National Yang-Ming University; Department of Critical Care Medicine, Taipei Veterans General Hospital, No. 201, ShiPai Rd., Taipei 112, Taiwan, R.O.C. Tel: +886 228757398, Fax: +886 228757579, e-mail: yylin@vghtpe.gov.tw

Key Words: Behavioral test, infarction, ischemic stroke, middle cerebral artery occlusion, rat. operation time, and has produced no obvious complications. Conclusion: This modified surgical technique may serve as a practical method for performing MCAO.

Stroke is a common disease that can have severe sequelae (1). About $80 \%$ of strokes are ischemic, and the most common infarction site is the middle cerebral artery (MCA) $(2,3)$. To facilitate progress in stroke research, translational research in animal models is essential in order to transfer basic research findings to the clinic. Researchers regularly choose the rodent model to create middle cerebral artery occlusion (MCAO) to simulate ischemic stroke (4-6). There exist several types of methods to induce MCAO, including intraluminal filament occlusion, craniotomy and direct MCA occlusion, and Endothelin-1-induced $\operatorname{MCAO}(7,8)$.

Among these methods, craniotomy is a common method, that has the advantage of allowing direct visualization of MCA through the surgical field (9). After the MCA has been exposed, it can be occluded by coagulation, transection, ligation or clips (7). However, the precise location of MCA occlusion is a critical issue. One study that used craniotomy in rats for MCAO revealed that occlusion of a more proximal part of the MCA led to larger infarct size (10). However, removing the cranial bone over the proximal MCA during craniotomy may be impeded by the zygomatic arch. If operators want to expose the most proximal part of the MCA, the zygomatic arch may be resected to provide adequate space $(11,12)$. However, this process may take time or affect the eating ability of rats. Other authors have exposed proximal MCA by displacing the brain but the large displacement of the brain may affect physiological functions (10). Intraluminal filament occlusion is another MCAO model that can mimic the clinical pathophysiology of stroke; 
however, this method will also obstruct anterior cerebral artery partially and produce striatum infarction (5). Thus, if researchers want to explore pure cortex ischemic injury without striatum invasion, it is not suitable to use intraluminal filament occlusion as an MCAO model.

Other than producing large and stable infarct size, a competent MCAO model is also expected to cause obvious neurological deficits thus simulating ischemic stroke. After ischemic brain injury, inflammatory cytokines such as tumor necrosis factor (TNF- $\alpha$ ), interleukin (IL)-1 $\beta$, and IL-6 are produced in the ischemic brain tissue and this neuroinflammation is a major mechanism in ischemic brain injury (13). The change of these cytokines were verified in vivo and in vitro. Thus, a satisfactory MCAO model should also produce these major changes in inflammatory cytokines.

In this study, a modified surgical method to expose proximal MCA was developed. Following craniotomy, the inferior edge of the craniotomy was enlarged with fine forceps. The method was easy to perform without significantly extending the operation time. Furthermore, this method did not lead to obvious complications such as reduced eating ability or mortality. Finally, the method was able to recapitulate significant effects of stroke, such as large and stable infarct volume, impaired behavior performance and significant production of inflammatory cytokines. Furthermore, the results of proximal and distal MCAO were also compared.

\section{Materials and Methods}

Animals. All animal experiments were approved by the Institutional Animal Care and Utilization Committee (IACUC) of National YangMing University, Taipei, Taiwan (approval ID: 1050315). Ten-weekold male Wistar Kyoto (WKY) rats weighing 200-300 g each were purchased from BioLASCO (Taipei, Taiwan). The rats were housed in the Laboratory Animal Center in National Yang-Ming University with a 12 -h light/dark cycle at a constant temperature $\left(25 \pm 2^{\circ} \mathrm{C}\right)$, and with unrestricted access to water and food.

Modified surgical method of MCAO. In order to improve the efficiency of MCAO, a modified surgical method was developed. Rats were anesthetized with an intraperitoneal injection of Zoletil $(30 \mathrm{mg} / \mathrm{kg})$ and Xylazine $(8 \mathrm{mg} / \mathrm{kg})$. A vertical incision was made on the midline of the neck, and the bilateral common carotid arteries (CCAs) were identified. After retracting the temporal muscle, a craniotomy was performed on the right temporal bone using a cutting burr (Figure 1a and b). During this period, the inferior edge of the hole was lowered as much as possible, until interrupted by the zygomatic arch. Then Dumont forceps (Roboz, Gaithersburg, MD, USA) were used to hold the residual bone on the inferior edge of the hole, with one tip between the dura and inner surface of cranial bone, and another tip on the outer surface of cranial bone (Figure 1c). After holding it with about $1 \mathrm{~mm}$ contact depth, the bone was pulled out piecemeal with a lateral and superior direction until the olfactory tract was exposed (Figure 1d). Then the dura matter was removed, and the MCA was permanently occluded by suture ligation at the level of the olfactory tract. Subsequently, bilateral CCA occlusion was performed by vessel clamp temporarily for $90 \mathrm{~min}$.

Another rat group was subjected to MCAO for purposes of comparison, using the conventional method. In this group, rats underwent the above procedure, but forceps were not used to remove the residual bone on the inferior edge of the hole. Thus, MCA was exposed to the level above the inferior cerebral vein and the distal MCA was permanently occluded by suture ligation above the inferior cerebral vein. Bilateral CCA occlusion was also performed temporarily for $90 \mathrm{~min}$. The sham group received identical anesthesia and surgical procedure without MCA and CCA occlusion. Rats that died during or after surgery were excluded for further experiment $(n=7)$.

Animal Behavioral Test. The rotarod test was utilized to evaluate motor coordination and balance alterations. Before MCAO was applied, rats were trained for running and balancing on the rotating drum (Rotamex-5, Columbus Instruments, Columbus, OH, USA) for 3 days. The rats deemed stable and that performed well on rotarod were assigned to groups that randomly received modified and conventional MCAO. At 1 ( $n=13 /$ group) and $3(n=7 /$ group) days after MCAO, the rotarod was accelerated from $4 \mathrm{rpm}$ to $40 \mathrm{rpm}$, with an acceleration of $1 \mathrm{rpm} / 10 \mathrm{sec}$. The latency before falling off the drum was recorded. Every rat received three trials for each training and testing day and the interval between each trial was 10 min. Successful trials after MCAO were defined as less than $75 \%$ running time on rod at 1 day after surgery, compared with preoperative performance.

Quantification of Infarct Volume. At 1 ( $\mathrm{n}=8 /$ group) and 3 ( $n=4 /$ group) days after MCAO, rats were anesthetized by intraperitoneal injection of Zoletil and Xylazine and transcardially perfused with phosphate-buffered saline (PBS). Their brains were expeditiously removed from the skull to determine infarction size, and then sliced into 2-mm-thick coronal sections. Then, they were stained with $2 \%$ 2,3,5- triphenyltetrazolium chloride (TTC; Sigma, St. Louis, MO, USA) solution for $15 \mathrm{~min}$ at room temperature without light, and then fixed with $4 \%$ paraformaldehyde. The unstained area represented the infarcted region, and the noninfarcted region revealed as red color on TTC-stained sections. To eliminate the effect of brain edema, the infarcted region was determined by subtracting the non-infarcted region in the ipsilateral hemisphere from that in the contralateral hemisphere. Infarct volumes were then calculated as the sum of the infarcted areas on each brain slice by using Image $\mathbf{J}$ software (National Institutes of Health, Bethesda, MD, USA).

Measurement of inflammatory cytokines. At 1 ( $n=4 /$ group) day after MCAO, rats were anesthetized by intraperitoneal injection of Zoletil and Xylazine and transcardially perfused with PBS. Their brains were removed and stored in $-80^{\circ} \mathrm{C}$ ultra-low temperature freezer. Then, the cerebral cortex of MCAO rats and sham group were homogenized and the levels of inflammatory cytokines (IL-1 $\beta$ and IL-6) were measured using commercially available ELISA kits (R\&D, Minneapolis, MN, USA).

Statistical analysis. Data are expressed as mean \pm the standard error of the mean (SEM). Categorical data were compared using chisquare test. Independent samples $t$-test was used to analyze the difference between two groups. All statistical analyses were 

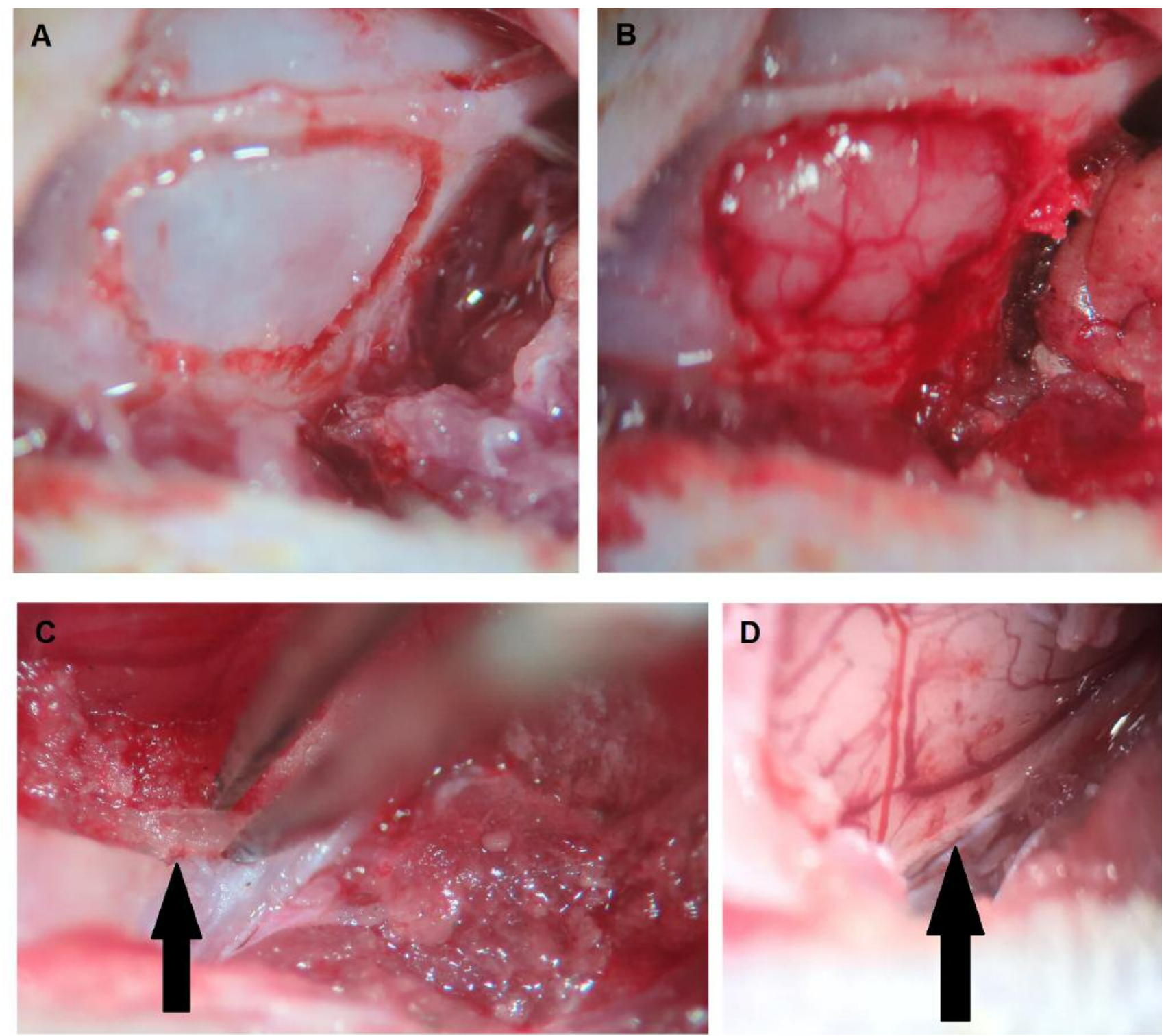

Figure 1. The procedures of the modified surgical method for middle cerebral artery occlusion (MCAO). (A) After skin incision and temporal muscle retraction, craniotomy contour was created using a burr tool. (B) Craniotomy and exposure of the underlying brain tissue. (C) Removal of the residual bone on the inferior edge of the craniotomy (arrow) using fine forceps. (D) Exposure of the middle cerebral artery at the level of olfactory branch (arrow) and preparation for MCAO.

performed using the SPSS 24.0 software package (SPSS, Inc, Chicago, IL, USA). Differences were considered significant at $p<0.05$.

\section{Results}

Operation time and mortality rate of the surgical procedures. The mean operation time from the point of skin incision to completion of a 90-minute CCA occlusion of rats receiving the modified and conventional method was $130.2 \mathrm{~min}$ and
121.4 min, respectively. Following MCAO, the mortality rates from the modified and conventional surgical methods were $9.4 \%$ and $13.3 \%$, respectively $(p=0.622)$. With the exception of the neurological deficits induced by MCAO, none of the rats had other obvious complications including eating difficulties.

The rats that received the modified surgical technique performed poorer in the rotarod test. Before MCAO, the rats receiving the modified and conventional surgical methods 

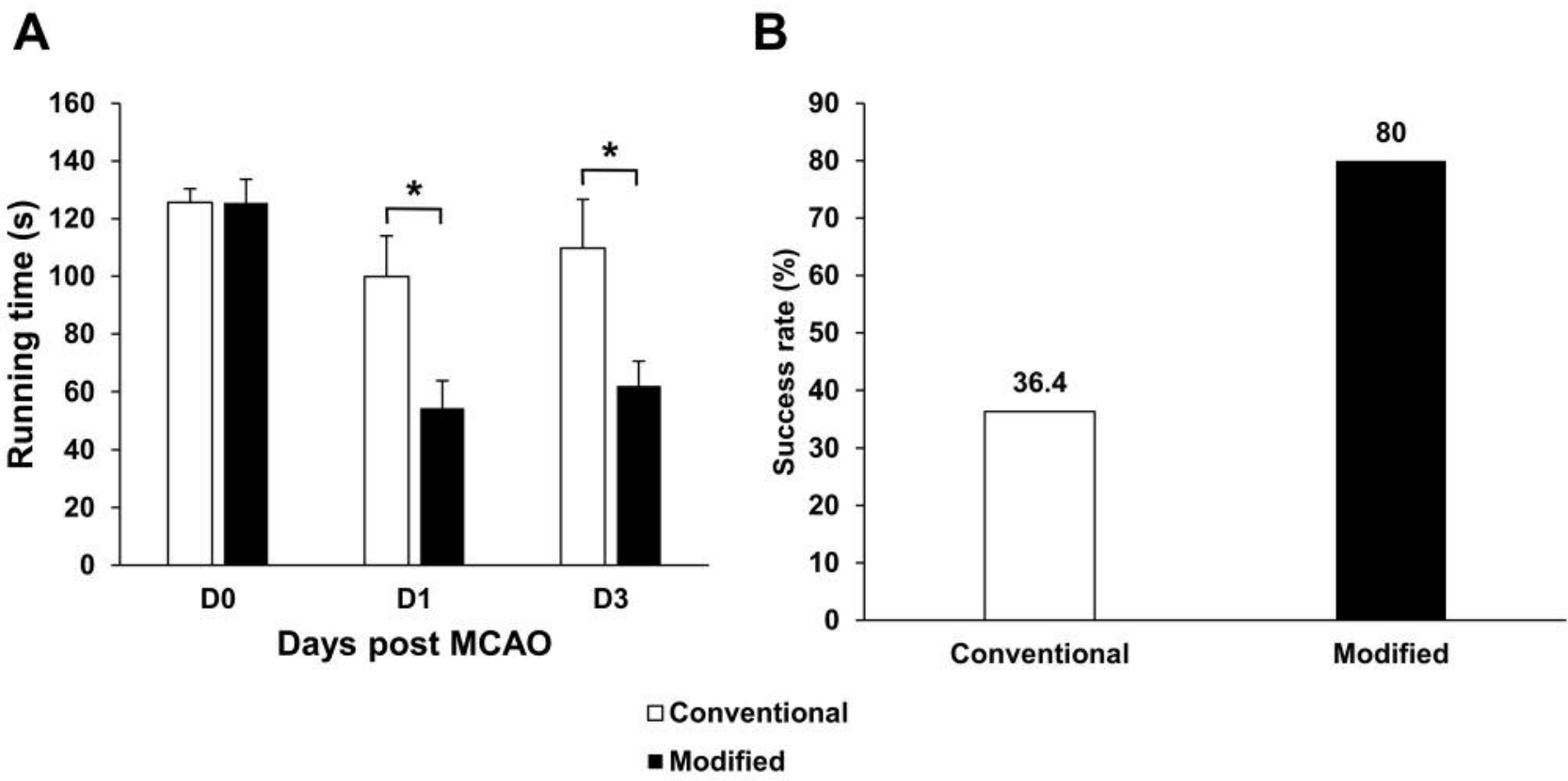

Figure 2. The modified surgical method produced poorer neurological function at 1 and 3 days after middle cerebral artery occlusion (MCAO). (A) Rotarod performance of MCAO rats receiving the modified or the conventional method. (B) The successful rates of MCAO in groups that received the modified or the conventional surgical procedure. $n=13 /$ group in 1 day and $n=7 /$ group in 3 days after MCAO. Data are presented as mean $\pm S E M . * p<0.05$.

had similar running times on the rod (126.1 sec vs. $125.6 \mathrm{sec}$, $p=0.930$ ) (Figure 2a). At 1 day after MCAO, the group the received modified surgical procedure had less running time on the rod, compared with the group that received the conventional surgical procedure (51.6 sec vs. $90.5 \mathrm{sec}$, $p=0.009$ ) (Figure 2a). At 3 days after MCAO, rats that received the modified method still stayed less time on the rod, compared with rats that received the conventional method (49.9 sec vs. $96.9 \mathrm{sec}, p=0.071)$ (Figure 2a). The group that received the modified surgical procedure had a success rate of $76.9 \%$, whereas the group that received the conventional surgical procedure had a success rate of only $46.2 \%$ (Figure $2 b$ ).

The modified surgical technique induced larger infarct volume. The ischemic region revealed as a pale color by TTC stain and involved only the cortex (Figure $3 \mathrm{a}$ and $\mathrm{b}$ ). At 1 day after MCAO, the infarct volume of the rats that received the modified surgical method was $189.5 \mathrm{~mm}^{3}$, larger than the $115.4 \mathrm{~mm}^{3}$ of the group that received the conventional surgical method $(p=0.001)$ (Figure $3 c)$. At 3 days after surgery, the infarct volumes in the groups operated with the modified and conventional methods were $144.6 \mathrm{~mm}^{3}$ and $102.3 \mathrm{~mm}^{3}$, respectively ( $p=0.004$ ) (Figure $3 \mathrm{c}$ ). With time, the infarct size became smaller in the group that received the modified surgical method $(p=0.021)$ (Figure 3c).
The modified surgical technique increased the levels of inflammatory cytokines. At 1 day after MCAO, the mean concentration of IL- $1 \beta$ in the ischemic cortex was 319.2 $\mathrm{pg} / \mathrm{ml}$, higher than the $44.3 \mathrm{pg} / \mathrm{ml}$ of the sham group $(p=0.039)$ (Figure 4). The mean concentration of IL-6 in the ischemic cortex was $1,024.1 \mathrm{pg} / \mathrm{ml}$ and in the sham group $70.9 \mathrm{pg} / \mathrm{ml}(p<0.001)$ (Figure 4).

\section{Discussion}

In the present study an efficient MCAO method via craniotomy in order to simulate ischemic cerebral cortex injury in rats is presented. Among the MCAO methods, intraluminal occlusion is a common method that requires skilled technique and expensive laser Doppler flowmetry to detect changes in MCA blood flow (14). It does not allow for direct observation of MCA. Besides, intraluminal occlusion usually induces infarction extending to striatum, so this occlusion method may not be suitable for studying exclusive cortex infarction. Alternatively, MCAO by way of craniotomy does not necessitate the use of expensive equipment, and can allow direct visualization of MCA. However, the site of MCAO significantly affects the results (10).

To induce larger infarct volume, the MCA must be occluded at the proximal site, such as the level of olfactory 
A 1 day after MCAO

Sham
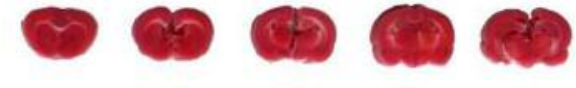

\section{Conventional}
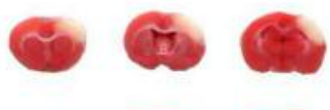

Modified
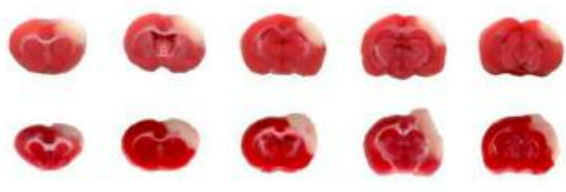

B

3 days after MCAO

Sham
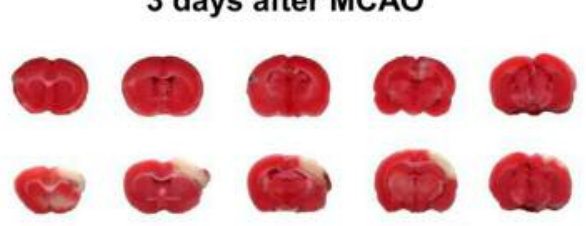

Conventional
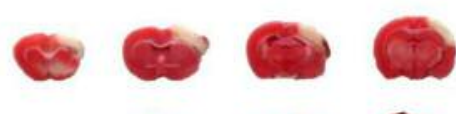

Modified
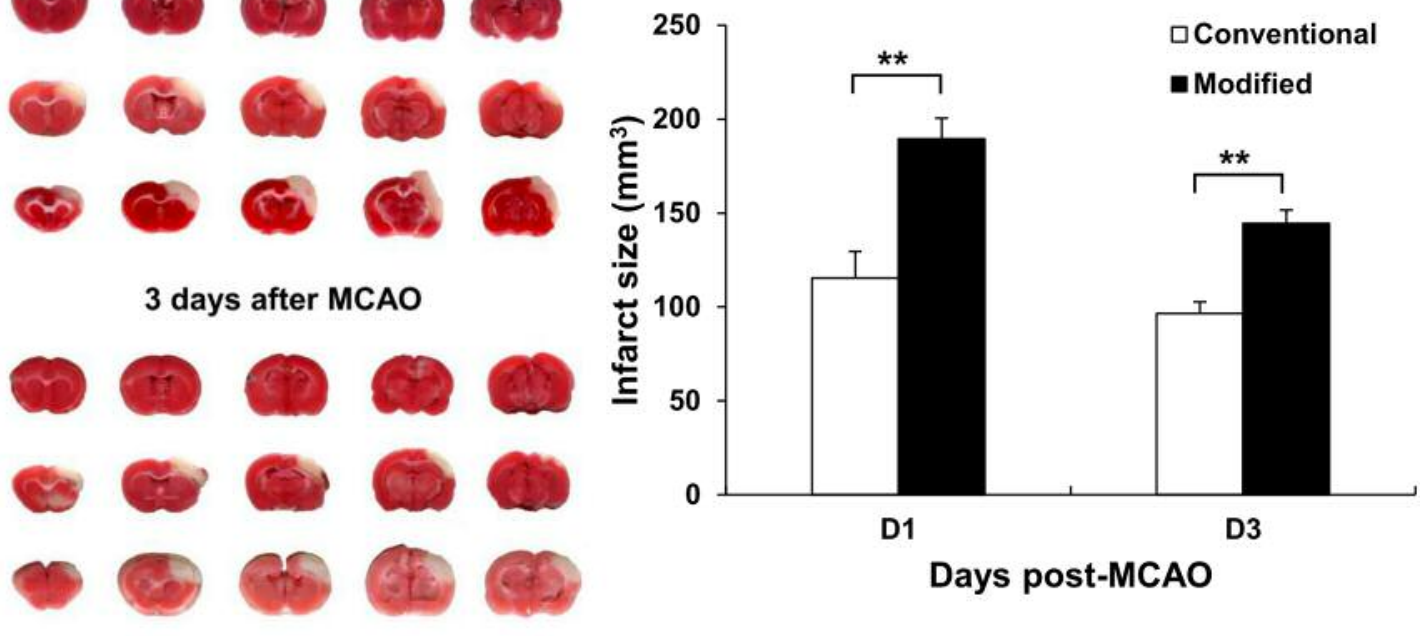

Figure 3. The modified surgical method produced larger infarct volume at 1 and 3 days after middle cerebral artery occlusion (MCAO). Representative, TTC stained brain slices from rats that received sham-operation, the modified surgical method or the conventional surgical method at (A) 1 day and (B) 3 days after MCAO. The pale region indicates the infarct area. (C) Quantification of infarct volume of rats that received the modified or conventional surgical method on 1 day and 3 days after MCAO. The sham-operated rats showed no infarction. $n=8 /$ group in 1 day and $n=4 /$ group in 3 days after MCAO. Data are presented as mean $\pm S E M . * * p<0.05$.
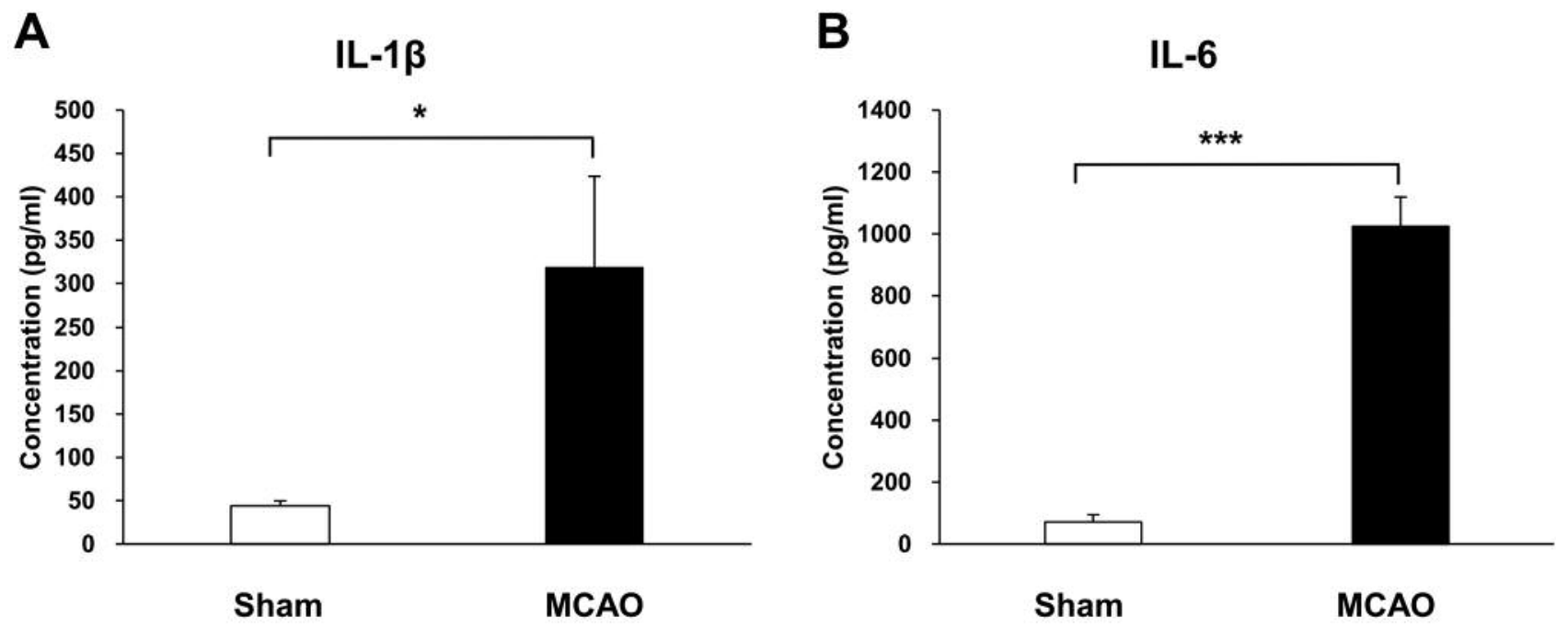

Figure 4. The modified surgical method produced higher levels of inflammatory cytokines in the ischemic cortex compared to the sham operation at 1 day after middle cerebral artery occlusion (MCAO). (A) The concentration of IL-1 $\beta$ in the cerebral cortex of MCAO and sham groups. (B) The concentration of IL-6 in the cerebral cortex of MCAO and sham groups, $n=4 /$ group. Data are presented as mean $\pm S E M . * p<0.05, * * * p<0.001$.

branch (10). However, if a burr tool is used to perform craniotomy, the burr shaft will be impeded by the zygomatic arch. Even if a burr with a specific angle is utilized, the procedure will still be impeded due to the width of the burr head. Removing the zygomatic arch is another option available to occlude the MCA at the proximal site. However, this option will likely extend the operation time, and may interfere with the eating ability of rodents. 
Thus, we have developed this novel and easy technique to improve the efficiency of MCAO by means of craniotomy. The key point in this modified procedure is to use fine forceps to remove the residual inferior cranial bone piecemeal, working in a lateral and superior direction. If a medial direction force is misapplied, the brain may be injured and the MCA could be impacted, potentially causing a hemorrhagic stroke. If an inferior direction force is misdirected, the residual bone cannot likely be efficiently removed. We suggest that forceps should be used to hold the bone with a $1 \mathrm{~mm}$ contact depth. If contact is shorter than this depth, the residual bone is difficult to remove; if longer than this depth, brain tissue injury may occur. This modified method can produce large infarct volume; however, the infarct area is confined to the cerebral cortex. Thus, this method may not be suitable when focusing on other areas such as striatum.

It is generally understood that bilateral CCA occlusion can increase the infarct volume due to additional block of the collateral circulation of the brain (15). If occluding the unilateral or bilateral CCA permanently, the mortality rate increases significantly (15). Chen et al. have reported an elevated mortality rate of $60 \%$ in rats receiving right MCA and bilateral CCA permanent occlusion (15). Thus, in this modified surgical procedure, temporal bilateral CCA occlusion was applied. This can increase the infarct volume without increasing the mortality rate.

Current studies focusing on the methodology of MCAO usually utilize infarct size and neurological deficits to evaluate if the MCAO model is successful. Changes in the cytokine levels are seldomly examined. Thus, to reinforce the evidence of a successful MCAO model, production of inflammatory cytokines was also measured and proved that our modified method increased the secretion of IL- $1 \beta$ and IL-6 in the ischemic cerebral cortex.

In conclusion, this study describes a modified surgical method to perform MCAO by way of craniotomy, with satisfactory results. This method simulates ischemic cerebral cortex injury, and produces large infarct volume and impaired motor and balance function. Besides, inflammatory cytokines were significantly increased in the ischemic region. Furthermore, further evidence indicated that that occlusion of the more proximal part of MCA by the modified surgical procedure can have a more severe stroke impact compared to the conventional method.

\section{Conflicts of Interest}

The Authors declare that they have no competing interests in regard to this study.

\section{Authors' Contributions}

Conceptualization: Chien-Fu Yeh, Tung-Yueh Chuang, Ming-Ying Lan and Yung-Yang Lin; Data curation: Chien-Fu Yeh, Tung-Yueh
Chuang, Yu-Wen Hung, Ching-Han Tsai, Hao-Xiang Huang and Yung-Yang Lin; Investigation: Chien-Fu Yeh and Yung-Yang Lin; Methodology: Tung-Yueh Chuang, Yu-Wen Hung, Ching-Han Tsai and Hao-Xiang Huang; Supervision: Ming-Ying Lan and YungYang Lin; Writing - original draft, Chien-Fu Yeh; Writing - review \& editing: Chien-Fu Yeh, Tung-Yueh Chuang, Ming-Ying Lan and Yung-Yang Lin.

\section{Acknowledgements}

This work was supported by the Ministry of Science and Technology of Taiwan (Grant number 105-2314-B-075-069-MY3, 106-2221-E-010-009, 106-3114-B-010-00, 107-2314-B-075-015MY2, 107-2321-B-039 -004 and 108-2643-F-008-003), and the Taipei Veterans General Hospital (Grant numbers V106D21-001MY2-1 and V107C-044 to Yung-Yang Lin; V108B-028 to ChienFu Yeh).

\section{References}

1 Casals JB, Pieri NC, Feitosa ML, Ercolin AC, Roballo KC, Barreto RS, Bressan FF, Martins DS, Miglino MA and Ambrosio CE: The use of animal models for stroke research: A review. Comp Med 61(4): 305-313, 2011. PMID: 22330245.

2 Sacco RL, Wolf PA and Gorelick PB: Risk factors and their management for stroke prevention: Outlook for 1999 and beyond. Neurology 53(7 Suppl 4): S15-24, 1999. PMID: 10532644.

3 Beal CC: Gender and stroke symptoms: A review of the current literature. J Neurosci Nurs 42(2): 80-87, 2010. PMID: 20422793.

4 Durukan A and Tatlisumak T: Acute ischemic stroke: Overview of major experimental rodent models, pathophysiology, and therapy of focal cerebral ischemia. Pharmacol Biochem Behav 87(1): 179-197, 2007. PMID: 17521716. DOI: 10.1016/j.pbb. 2007.04.015

5 Fluri F, Schuhmann MK and Kleinschnitz C: Animal models of ischemic stroke and their application in clinical research. Drug Des Devel Ther 9: 3445-3454, 2015. PMID: 26170628. DOI: 10.2147/DDDT.S56071

6 Liu F and McCullough LD: Middle cerebral artery occlusion model in rodents: Methods and potential pitfalls. J Biomed Biotechnol 2011: 464701, 2011. PMID: 21331357. DOI: 10.1155/2011/464701

7 Howells DW, Porritt MJ, Rewell SS, O'Collins V, Sena ES, van der Worp HB, Traystman RJ and Macleod MR: Different strokes for different folks: The rich diversity of animal models of focal cerebral ischemia. J Cereb Blood Flow Metab 30(8): 1412-1431, 2010. PMID: 20485296. DOI: 10.1038/jcbfm.2010.66

8 Lee S, Hong Y, Park S, Lee SR, Chang KT and Hong Y: Comparison of surgical methods of transient middle cerebral artery occlusion between rats and mice. J Vet Med Sci 76(12): 1555-1561, 2014. PMID: 25649935. DOI: 10.1292/jvms.140258

9 Wayman C, Duricki DA, Roy LA, Haenzi B, Tsai SY, Kartje G, Beech JS, Cash D and Moon L: Performing permanent distal middle cerebral with common carotid artery occlusion in aged rats to study cortical ischemia with sustained disability. J Vis Exp 108: 53106, 2016. PMID: 26967269. DOI: $10.3791 / 53106$ 
10 Bederson JB, Pitts LH, Tsuji M, Nishimura MC, Davis RL and Bartkowski H: Rat middle cerebral artery occlusion: Evaluation of the model and development of a neurologic examination. Stroke 17(3): 472-476, 1986. PMID: 3715945.

11 Tamura A, Graham DI, McCulloch J and Teasdale GM: Focal cerebral ischaemia in the rat: 1 . Description of technique and early neuropathological consequences following middle cerebral artery occlusion. J Cereb Blood Flow Metab 1(1): 53-60, 1981. PMID: 7328138. DOI: 10.1038/jcbfm.1981.6

12 Theodorsson A, Holm L and Theodorsson E: Modern anesthesia and peroperative monitoring methods reduce per- and postoperative mortality during transient occlusion of the middle cerebral artery in rats. Brain Res Brain Res Protoc 14(3): 181190, 2005. PMID: 15795172. DOI: 10.1016/j.brainresprot. 2005.01.002

13 Lambertsen KL, Biber K and Finsen B: Inflammatory cytokines in experimental and human stroke. J Cereb Blood Flow Metab 32(9): 1677-1698, 2012. PMID: 22739623. DOI: 10.1038/ jcbfm.2012.88
14 Schmid-Elsaesser R, Zausinger S, Hungerhuber E, Baethmann A and Reulen HJ: A critical reevaluation of the intraluminal thread model of focal cerebral ischemia: Evidence of inadvertent premature reperfusion and subarachnoid hemorrhage in rats by laser-doppler flowmetry. Stroke 29(10): 2162-2170, 1998. PMID: 9756599.

15 Chen ST, Hsu CY, Hogan EL, Maricq H and Balentine JD: A model of focal ischemic stroke in the rat: Reproducible extensive cortical infarction. Stroke 17(4): 738-743, 1986. PMID: 2943059.

Received March 21, 2019

Revised April 23, 2019

Accepted April 24, 2019 